\title{
Modification of Wood with Monoethanolamino- borate by The Data of X-Ray Photoelectron Spectroscopy
}

\author{
Irina Stepina ${ }^{1, *}$, and Irina Kotlyarova ${ }^{2}$ \\ ${ }^{1}$ Moscow State University of Civil Engineering, Yaroslavskoye shosse, 26, Moscow, 129337, Russia \\ ${ }^{2}$ Bryansk State Technical University, Boulevard 50 let Oktyabrya, 7, Bryansk, 241035, Russia
}

\begin{abstract}
The difficulty of wood protection from biocorrosion and fire is due to the fact that modifiers in use are washed out from the surface of the substrate under the influence of environmental factors. This results in a rapid loss of the protective effect and other practically important wood characteristics caused by the modification. To solve this problem is the aim of our work. Here, monoethanolaminoborate is used as a modifier, where electron-donating nitrogen atom provides a coordination number equal to four to a boron atom, which determines the hydrolytic stability of the compounds formed. Alpha-cellulose ground mechanically to a particle size of $1 \mathrm{~mm}$ at most was used as a model compound for the modification. X-ray photoelectron spectra were recorded on the XSAM-800 spectrometer (Kratos, UK). Prolonged extraction of the modified samples preceded the registration of the photoelectron spectra to exclude the fixation of the modifier molecules unreacted with cellulose. As a result of the experiment, boron and nitrogen atoms were found in the modified substrate, which indicated the hydrolytic stability of the bonds formed between the modifier molecules and the substrate. Therefore monoethanolaminoborate can be considered as a non-extractable modifier for wood-cellulose materials.
\end{abstract}

\section{Introduction}

The difficulty of wood protection from biocorrosion and fire is due to the fact that modifiers in use are washed out from the surface of the substrate under the influence of environmental factors. This results in a rapid loss of the protective effect and other practically important wood characteristics caused by the modification. Our work is aimed at solving this problem. Boron compounds are widely used for preservation and fire hardening of wood-cellulose materials $[1,2]$.

Chemical bonds formed between the hydroxyl groups and the trivalent boron compounds are hydrodynamically unstable, easily destroyed, and the modifier is washed out from the surface of the wood [3]. One relatively new approach is the development of protein-based boron-containing preservatives. Protein- and boron-containing preservatives such as albuminborate significantly slow down the extraction of boron from the treated

\footnotetext{
* Corresponding author: sudeykina@mail.ru
} 
wood $[4,5]$. Accelerated biological tests have shown that boric acid partly reacts to wood due to formation of salt with albumin and other proteins which become insoluble after thermal coagulation. Durability of protection by such antiseptics can be compared to the CCA preservatives containing copper, chrome and arsenic. Boron-containing protein-based preservatives are completely safe for humans and have a wide spectrum of action. Investigations of the chemical mechanisms of interaction of boric acid with proteins have shown that the formation of salt is not completely irreversible, since the content of free boric acid in the system increases with time, and there is a tendency to transition of the "boric acid-protein complex" system into the equilibrium state that is characterized by a certain ratio of components, different for each specific protein [4].

To determine the type of particles present in the solution, the 11B NMR spectra of reaction products of boric acid and albumin in water were studied [6]. In acidic medium $\left(\mathrm{pH}<7\right.$ ), only non-dissociated molecules of $\mathrm{H}_{3} \mathrm{BO}_{3}$ were present, which corresponds to a chemical shift of $20 \mathrm{ppm}$. In the alkaline medium $(\mathrm{pH}>7)$ signals characteristic of uncharged and charged boron atoms appear in the range of $16 \mathrm{ppm}$ and 12.6-13.7 ppm, indicating the presence of three ionic species $\left[\mathrm{B}(\mathrm{OH})_{4}\right]^{-},\left[\mathrm{B}_{3} \mathrm{O}_{3}(\mathrm{OH})_{4}\right]^{-},\left[\mathrm{B}_{3} \mathrm{O}_{3}(\mathrm{OH})_{5}\right]^{2-}$ in the solution. The signal in the range of 1.7-1.8 ppm points to the presence of the interaction product of polyborate ion and the protein. The heating of the solution leads to a relative increase in the fraction of the last two signals indicating both an increase in the number of charged particles in the solution and an increase in the proportion of boron ions reacting with the protein. This results in a varying efficiency of albuminborate and a varying degree of the preservative extraction, depending on $\mathrm{pH}$ and reaction conditions, which was confirmed by laboratory and field tests [6].

We suggest that the use of four-coordinate boron compounds can be a solution to the problem of the modifier extraction from the substrate surface. It is known that compounds containing four-coordinate boron atom are sufficiently resistant to hydrolysis [7]. Among them is monoethanolaminoborate containing the electron-donating nitrogen atom that provides a coordination number equal to four to a boron atom.

\section{Methodology}

Monoethanolaminoborate was synthesized using $\mathrm{H}_{3} \mathrm{BO}_{3}$ (reagent grade) and monoethanolamine (special purity grade). The reaction was carried out at room temperature either in solution or between pure substances. The product of reaction between pure $\mathrm{H}_{3} \mathrm{BO}_{3}$ and monoethanolamine consisted of two phases: crystalline and gel-like. The phases were separated and dissolved in water. Interesting to note that the reaction between boric acid and monoethanolamine is quite intensive and exothermic. The opinions of scientists on the product of this reaction were divided. Some researches [8] suggest that the reaction product contains ionic bond. Other researches $[9,10]$ suggest that a compound with the coordinate bond $\mathrm{B} \leftrightarrow \mathrm{N}$ is formed. In this case the reaction can be illustrated as in figure 1 .

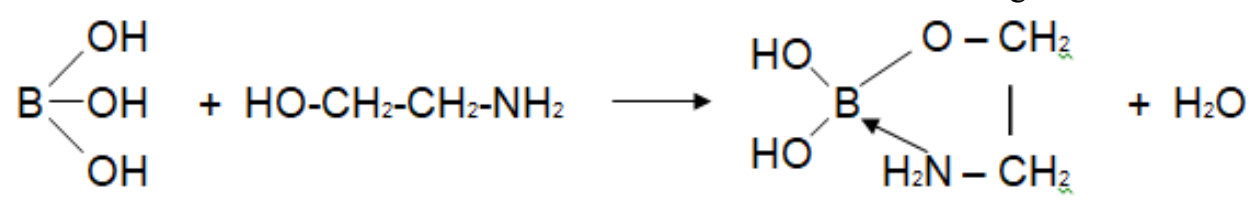

Fig. 1. The reaction of boric acid with monoethanolamine.

As the model compound for the modification, $\alpha$-cellulose mechanically ground to a particle size of $1 \mathrm{~mm}$ at most and 50\% solutions of modifiers were used: a solution obtained from the gel phase (sample 1); a solution obtained from the crystalline phase (sample 2); a solution obtained by mixing the starting materials (sample 3 ). The reaction was carried out 
at room temperature for three hours. The modified samples were extracted with distilled water for three hours to remove the excess the modifier.

X-ray photoelectron spectra were recorded on the XSAM-800 spectrometer (Kratos, $\mathrm{UK})$. The magnesium anode with the characteristic radiation energy $\mathrm{MgK}=1253.6 \mathrm{eV}$ was used as an excitation source. The power released at the anode during the recording of the spectra did not exceed $90 \mathrm{~W}$. The background caused by the secondary electrons and photoelectrons lost energy was approximated by a straight line. The measurements were carried out at a pressure of $\sim 5 \cdot 10^{-8} \mathrm{~Pa}$. The samples were mounted on a titanium holder using a double-sided adhesive tape. The spectra were recorded at room temperature, the depth of the investigated surface was $100 \AA$. The spectrometer was calibrated by the $\mathrm{Au}$ $4 \mathrm{f}_{7 / 2}$ and $\mathrm{Ni} 2 \mathrm{p}_{3 / 2}$ peaks which energies corresponded to 84.0 and $852.7 \mathrm{eV}$, respectively. The quantitative analysis was performed on the basis of equation (1):

$$
I=A(E) \sigma f n \lambda(E)
$$

Where $\mathrm{A}(E)$ is the point spread function of the spectrometer, $\sigma$ is the photoionization crosssection of the inner shell, $\mathrm{f}$ is the asymmetry parameter, $\mathrm{n}$ is the number of atoms per unit volume, $\lambda$ is the free path of photoelectrons without inelastic energy loss. The product $\sigma \mathrm{f} \lambda$ is called the element sensitivity coefficient (ESC). For the given energy range, the functions $\mathrm{A}(E)$ and $(E)$ are proportional to $\mathrm{E}^{3 / 2}$ and $\mathrm{E}^{1 / 2}$, respectively. The numerical values of $\sigma$ and $\mathrm{f}$ for determination of the ESC were taken from [11]. Compensation of the surface charge was carried out using peak $\mathrm{O} 1 \mathrm{~s}$, to which an energy of $532.9 \mathrm{eV}$ was attributed [12].

\section{Results and discussion}

Characteristic peaks for $\mathrm{B}$ and $\mathrm{N}$ atoms are not visible in the panoramic photoelectron spectra of all three samples. This can be explained by the presence of surface contaminants which can significantly reduce the intensity of the peaks of the elements being investigated. It is also necessary to take into account that photoelectron emission from a non-conductive sample (cellulose) which surface is charged positively leads to an energy barrier formation, and photoelectrons spend additional energy for overcoming this barrier. However, when the photoelectron spectra are considered in more detail (figures 2 and 3), peaks characteristic for $\mathrm{B}$ and $\mathrm{N}$ atoms appear.

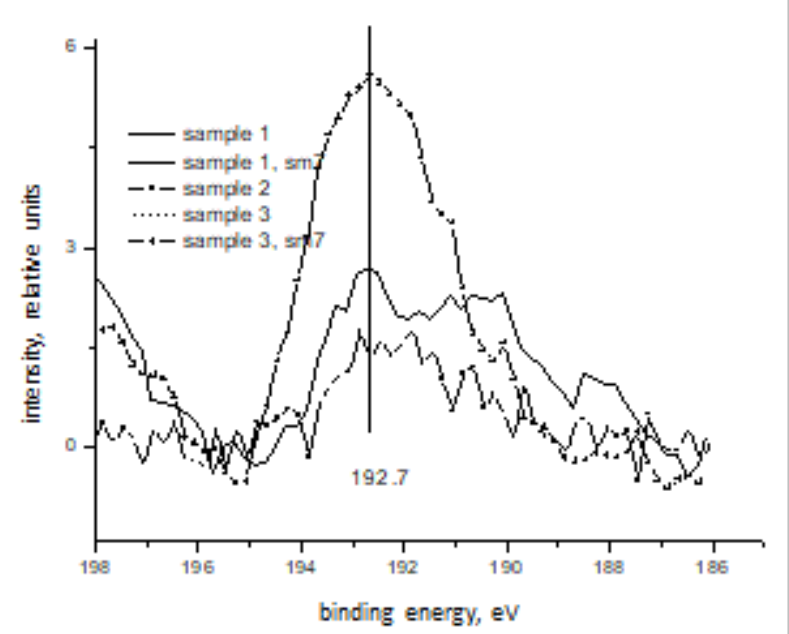

Fig. 2. Photoelectron spectra B 1s of samples 1-3, smoothed over seven points. 


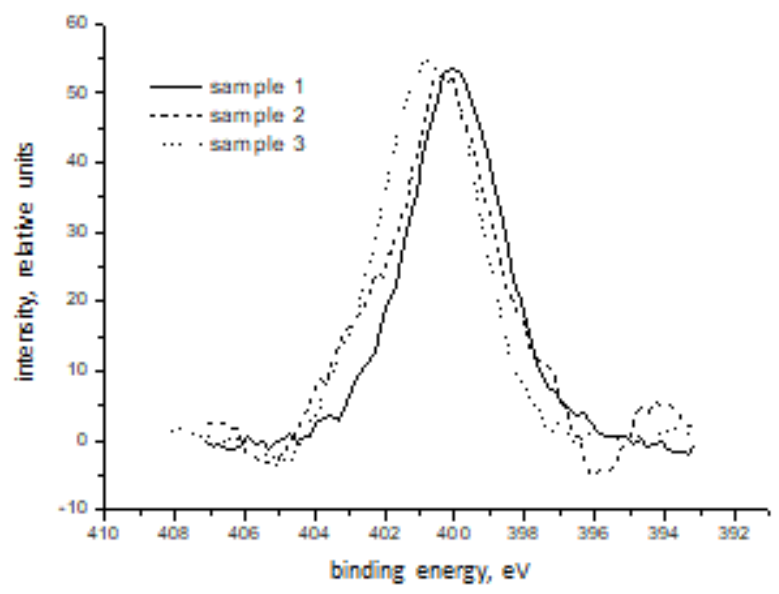

Fig. 3. Photoelectron spectra N 1s of samples 1-3, smoothed over seven points.

Under close examination of the spectral peaks characteristic for $\mathrm{O}$ and $\mathrm{C}$, figures 4 and 5 , one can notice the appearance of a new asymmetry in comparison to the peaks of unmodified cellulose [12]. This is especially true for samples 2 and 3. The appearance of asymmetry means the formation of new bonds between the $\mathrm{O}$ - and $\mathrm{B}$ - atoms, C- and $\mathrm{O}-$ atoms, which points to the chemical interaction of the modifier and the substrate.

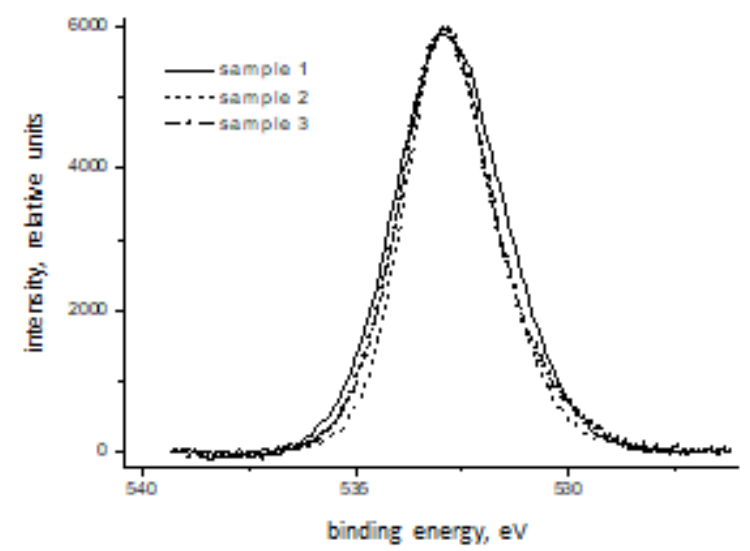

Fig. 4. Photoelectron spectra O 1s of samples 1-3

Smoothed over seven points photoelectron spectra of $\mathrm{B}$ and $\mathrm{N}$ atoms in the modified cellulose composition are presented in figures 2 and 3 . The obtained values of bond energy for B-N, B-O and values of bond energy of B with neighboring atoms available from literature $[13,14]$ are in Table 1 . It can be seen from the table that the obtained values for B bond energies do not correspond to values from any literary source in which these values were calculated for the three-coordinate $\mathrm{B}$ atom. This gives grounds to assume that in our case the boron atom may be four-coordinate, and, consequently, $\mathrm{N}$ is also four-coordinate, which serves as a confirmation of the formation of the donor-acceptor bond between $\mathrm{B}$ and $\mathrm{N}$ atoms, according to the scheme presented in figure 1. 


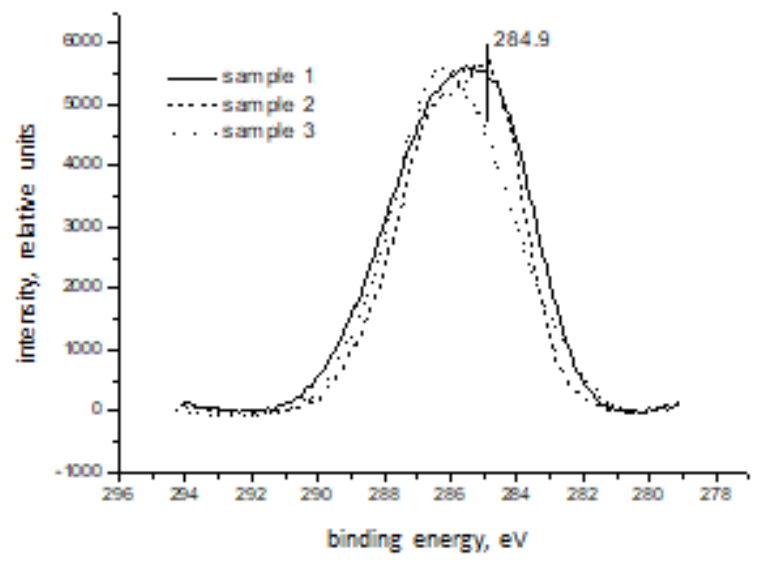

Fig. 5. Photoelectron spectra C 1s of samples 1-3.

Then the interaction between the hydroxyl groups of cellulose and monoethanolamineborate can be represented as diagram, figure 6 . This hypothesis is also confirmed by the fact that, even after prolonged extraction with water (for 3 hours), the boron and nitrogen atoms were retained in the cellulose indicating the formation of strong hydrolytically stable chemical bonds.

Table 1. The values of bond energies

\begin{tabular}{|l|l|l|l|l|l|l|l|}
\hline & B 1s & N 1s & O 1s & N1s-B1s & B1s-O1s & $\begin{array}{l}\text { Handbook } \\
{[13]}\end{array}$ & $\begin{array}{l}\text { NIST B1s } \\
{[14]}\end{array}$ \\
\hline $\begin{array}{l}\text { Sample } \\
1\end{array}$ & 191.6 & 400.1 & 533.03 & 208.5 & 341.43 & $\begin{array}{l}\text { BN: 190.7, } \\
398.3\end{array}$ & $190.5,398.2$ \\
\hline $\begin{array}{l}\text { Sample } \\
2\end{array}$ & 192.2 & 400.3 & 532.86 & 208.1 & 340.66 & $\mathrm{~B}_{2} \mathrm{O}_{3}: 193.4$ & $193.6,533.2$ \\
\hline $\begin{array}{l}\text { Sample } \\
3\end{array}$ & 192.6 & 400.8 & 532.86 & 208.2 & 340.26 & BOH: 193.2 & $\begin{array}{l}\text { H3BO3: } \\
193.4\end{array}$ \\
\hline
\end{tabular}

From the data of elemental analysis of modified cellulose obtained by the XPS method, Table 2, the smallest quantity of B is contained in the first sample. This gives grounds to consider that the gel obtained by mixing of pure $\mathrm{H}_{3} \mathrm{BO}_{3}$ and monoethanolamine is predominantly the remains of unreacted substances mixture. The highest content of $\mathrm{B}$ was noted in the third sample, Table 2. It can therefore be concluded that the most effective solution for cellulose modification is the solution obtained at the stage of the reaction of the starting substances.

Table 2. Quantitative analysis of modified cellulose by XPS

\begin{tabular}{|l|l|l|l|}
\hline & № 1 & № 2 & № 3 \\
\hline Elmt & c, at. \% & c, at. \% & c, at. \% \\
\hline C1s & 68.40 & 67.99 & 65.90 \\
\hline O1s & 31.22 & 31.15 & 33.08 \\
\hline N1s & 0.34 & 0.46 & 0.54 \\
\hline B1s & 0.04 & 0.40 & 0.48 \\
\hline
\end{tabular}




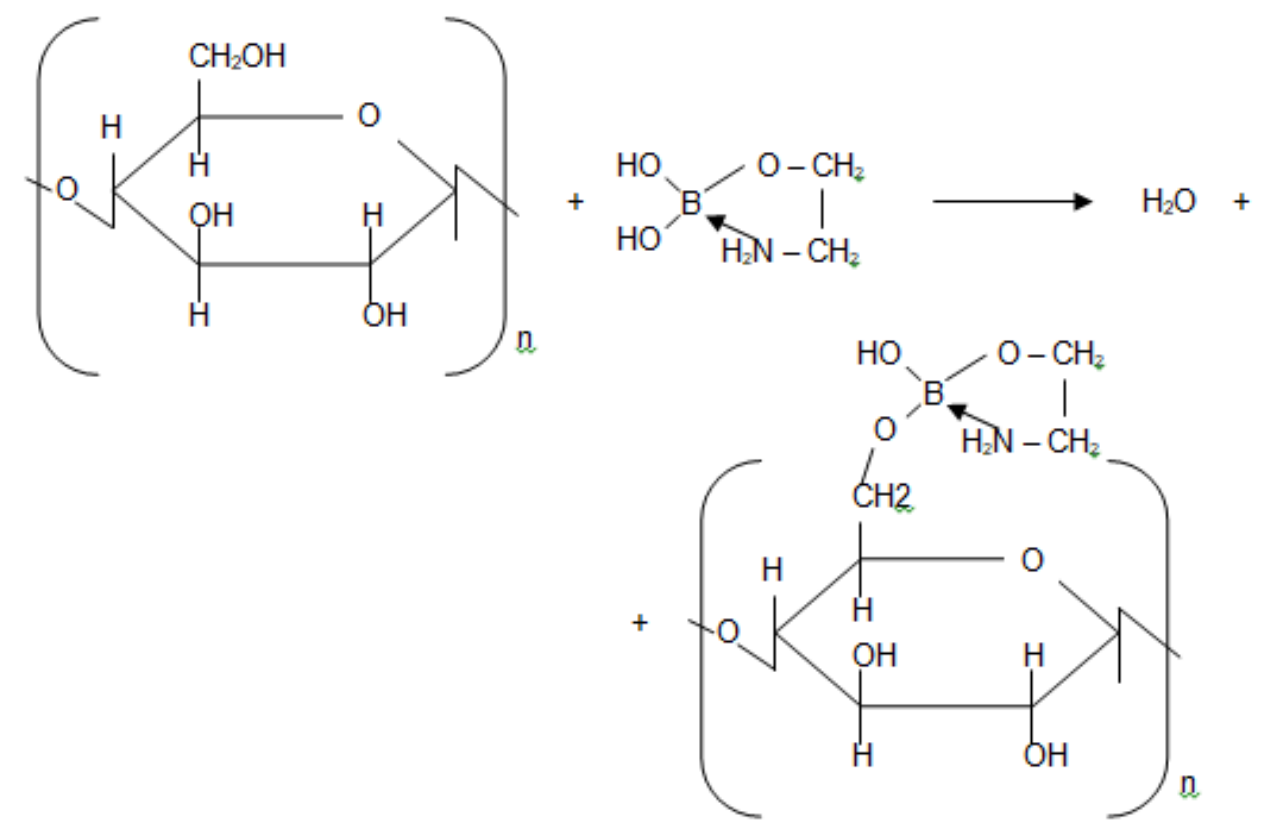

Fig. 6. The reaction of cellulose with monoethanolaminoborate

Thus, based on the experimental data obtained by X-ray photoelectron spectroscopy of the modified wood cellulose samples, it was established that monoethanolamine borate reacts chemically with hydroxyl groups of cellulose to form strong hydrolytically stable bonds. In practice, non-extractability of the modifier from the wood surface for a long period of operation provides building structures with durable protection against biocorrosion and fire.

\section{References}

1. N.A. Ermush Boron-containing preservatives and flame retardants for wood protection. Riga, LatNIINTI, 63 (1988)

2. V.D. Skorokhodov Protection of nonmetallic construction materials from biocorrosion. Moscow: Vysshaya shkola, 204 (2004)

3. N.G. Butylkina, N.I. Bekasova and others Khimija drevesiny, 5, 20-24 (1982)

4. M.F. Thevenon, A. Pizzi, J.P. Haluk Holz als Roh - und Werkstoff, 55 (5), 293-296 (1997)

5. M.F. Thevenon, A. Pizzi, J.P. Haluk Holzforschung, 52 (3), 241-248 (1998)

6. M.F. Thevenon, A. Pizzi, Holz als Roh - und Werkstoff, 61 (6), 457-464 (2003)

7. E.P. Alekperov, A.M. Reznik Boron complexes: synthesis, application. Moscow: MSU, 208 (2000)

8. V.G. Skvortsov, A.K. Molodkin and others Russian Journal of Inorganic Chemistry, 25, 1964-1969 (1980)

9. K. Nidentsu, J. Dawson Chemistry of boron-nitrogen compounds. Moscow: Mir, 234 (1968)

10. E.R. Alekperov, A.M. Reznik Koordinatsionnja Khimija, 19 (1), 5-14 (1993)

11. I.M. Band, Yu.I. Kharitonov, M.B. Trzhaskovskaya Atomic Data and Nuclear Data Tables, 23, 443 (1979) 
12. G. Beamson, D. Briggs High Resolution XPS of Organic Polymers: The Scienta ESCA 300 Database. Chichester: Wiley. (1992)

13. C.D. Wagner, W.M. Riggs and other Handbook of x-ray photoelectron spectroscopy by. Perkin-Elmer corporation physical electronics division, (1978)

14. C.D. Wagner, A.V. Naumkin, A. Kraut-Vass, J.W. Allison, C.J. Powell NIST Standard Reference Database 20,Version 3.4. Web Vers: http://srdata.nist.gov@xps (2004)

15. A.O. Adamtsevich, A.P. Pustovgar, , S.A. Pashkevich, A.V. Eremin, International Journal of Applied Engineering Research, 11(3), 1609 (2016)

16. S. Pashkevich, A. Pustovgar, A. Adamtsevich, A. Eremin, Applied Mechanics And Materials, 584-586, 1659 (2014) 